\title{
Gravidez e parto após embolização arterial para tratamento de leiomioma uterino
}

\author{
Pregnancy and delivery after arterial embolization for fibroid treatment
}

Cláudio Emilio Bonduki ${ }^{1}$, Patrícia Gonçalves², Cláudio Yokohama ${ }^{3}$, Odon Ferreira da Costa Edmund Chada Baracat ${ }^{5}$, Geraldo Rodrigues de Lima ${ }^{5}$, Manoel João Batista Castello Girão ${ }^{6}$

\section{RESUMO}

Objetivo: Analisar a evolução da gestação e partos após tratamento de leiomioma uterino por embolização das artérias uterinas. Métodos: Foram incluídas na avaliação inicial 112 pacientes submetidas a embolização de artérias uterinas para tratamento de mioma uterino. Destas, somente nove desejavam o tratamento conservador para manter a capacidade reprodutiva. Este procedimento foi indicado para estas nove pacientes, pois elas não eram susceptíveis ao tratamento conservador cirúrgico. Submeteram-se a embolização das artérias uterinas com partículas de álcool polivinílico ou embosferas com diâmetro de 500 a $700 \mu \mathrm{m}$ e evoluíram sem intercorrências. Resultados: Durante o acompanhamento dessas nove pacientes houve boa resposta clínica, com redução significativa no volume do útero e dos miomas. Dessas nove, quatro engravidaram, sendo que duas tiveram abortamento precoce e duas evoluíram normalmente até o final da gestação com parto a termo, sendo um deles gemelar. Conclusão: A embolização de artérias uterinas é uma opção para o tratamento de miomas uterinos e apresenta bons resultados clínicos e anatômicos, permitindo manter a capacidade reprodutiva.

PALAVRAS-CHAVE: Mioma; Neoplasias uterinas; Leiomioma/terapia; Embolização terapêutica/métodos; Fertilidade; Gravidez

\section{ABSTRACT}

Purpose: To analyze gestation evolution and deliveries after myoma treatment by embolization of the uterine arteries. Methods: In the initial evaluation, 112 patients submitted to embolization of uterine arteries were included for treatment of myoma. From those, only nine wanted to be submitted to conservative treatment in order to keep their reproductive capacity. This procedure was indicated to the nine patients, since they were not susceptible to a conservative surgical treatment. They were submitted to embolization of the uterine arteries with particles of polyvinyl alcohol or embospheres with diameters ranging from 500 to $700 \mu \mathrm{m}$, and they have evolved without intercurrence. Results: During the follow-up of these patients, there was a good clinical response with significant reduction in the uterus and myoma volumes. Four of them got pregnant, two had an early abortion and two evolved normally till the end of gestation with a term delivery. One of these had twins. Conclusion: Embolization of the uterine arteries is an option for the treatment of uterine myoma, and presents good clinical and anatomical results, allowing patients to preserve their reproductive capacity.

KEYWORDS: Myoma; Uterine neoplasms; Leiomyoma/therapy; Embolization, therapeutic/methods; Fertility; Pregnancy

Departamento de Ginecologia, Escola Paulista de Medicina, Universidade Federal de São Paulo - UNIFESP - São Paulo (SP), Brasil.

1 Professor Assistente, Departamento de Ginecologia, Escola Paulista de Medicina, Universidade Federal de São Paulo - UNIFESP - São Paulo (SP), Brasil.

2 Ginecologista, Hospital Sírio Libanês - São Paulo (SP), Brasil.

3 Radiologista Intervencionista, Departamento de Imagem, Escola Paulista de Medicina, Universidade Federal de São Paulo - UNIFESP - São Paulo (SP), Brasil.

4 Chefe do Departamento de Radiologia Vascular, Hospital Sírio Libanês - São Paulo (SP), Brasil.

5 Professor Titular, Departamento de Ginecologia, Escola Paulista de Medicina, Universidade Federal de São Paulo - UNIFESP - São Paulo (SP), Brasil.

6 Professor Livre-docente, Departamento de Ginecologia, Escola Paulista de Medicina, Universidade Federal de São Paulo - UNIFESP - São Paulo (SP), Brasil.

Correspondência: Cláudio Emilio Bonduki

Recebido em: 22/08/2005

Aceito com modificações em: 13/11/2006

Rev Bras Ginecol Obstet. 2006; 28(10): 596-600. 


\section{Introdução}

O início no emprego da embolização de artérias uterinas em casos de leiomioma uterino tinha como finalidade reduzir o tamanho e vascularização dos tumores no pré-operatório de miomectomia ou histerectomia, para facilitar o procedimento cirúrgico e evitar maior sangramento e outras complicações. No entanto, observou-se que havia ótimo controle das alterações clínicas (sangramento, algia pélvica e sintomas compressivos) e significante redução no tamanho do útero e dos miomas após o procedimento, tornando dispensável o tratamento cirúrgico complementar na grande maioria das pacientes. Em 1995 houve a primeira publicação do emprego desta terapêutica para mioma uterino ${ }^{1}$ com posterior disseminação deste tratamento para centenas de centros em todo o mundo.

Inicialmente, temia-se realizar embolização arterial em pacientes desejosas de manter a fertilidade, pelo receio de seu impacto sobre a capacidade reprodutiva, especialmente eventuais complicações após o procedimento, como infecção pós-necrose maciça do útero ou insuficiência ovariana.

Miomas sofrem necrose asséptica após embolização, podendo eventualmente se infectar. $\mathrm{Na}$ grande maioria dos relatos, a infecção ocorre com maior freqüência em miomas submucosos (especialmente pediculados) ou com porção submucosa predominante, associada a uma avaliação prévia inadequada da flora vaginal e endocervical. Necrose maciça do útero também é outro fator de risco para infecção do órgão. Esta eventualidade pode ocorrer quando há obstrução no tronco da artéria uterina durante o procedimento de embolização. Entretanto, a ocorrência de infecção é baixa, < 0,7\% dos casos; atualmente, devido aos cuidados na seleção das pacientes, melhor preparo pré-operatório, técnica e materiais adequados, a incidência é ainda menor ${ }^{2}$.

Insuficiência ovariana pode ocorrer em 0,7\% das pacientes submetidas a embolização, sendo que a grande maioria é transitória. A ocorrência de insuficiência definitiva é mais freqüente em mulheres com mais de 45 anos.\# Os fatores que podem promover esta complicação dependem muito da técnica e de materiais inadequados ${ }^{2}$.

Diante da preocupação em manter o potencial reprodutivo após a embolização arterial, houve modificação em alguns pontos relacionados com procedimento e materiais empregados. Assim, atualmente há indices de complicação mínimos, que permitem manter a fertilidade; isto está documentado em trabalhos sobre gestação e parto após este tratamento ${ }^{3,4}$.

O objetivo deste artigo é revisar a literatura, apresentando nossa experiência na embolização de artérias uterinas em pacientes que desejavam engravidar após o tratamento para mioma uterino.

\section{Métodos}

Iniciamos os tratamentos para leiomioma por embolização arterial em maio de 2000, tendo o procedimento sido realizado em 112 casos até outubro de 2004. A média de idade das pacientes foi de 38 anos ( 27 a 49 anos) e a raça branca foi a mais freqüente $(81,2 \%)$.

As principais indicações foram alteração menstrual $(59,3 \%)$, algia pélvica $(43,7 \%)$, aumento progressivo no tamanho do útero e mioma $(78,1 \%)$, sintomas compressivos $(23,1 \%)$, falha no tratamento conservador prévio (recidiva de miomectomia em $21,8 \%$ e falha no tratamento clínico em 31,2\%), dificuldade no acesso cirúrgico $(37,5 \%)$, desejo de manter o útero $(78,1 \%)$, opção não cirúrgica $(68,7 \%)$ e infertilidade $(8,0 \%)$.

A técnica utilizada foi a de Seldinger e consiste na punção da artéria femural direita. Inicialmente um cateter é introduzido através desta artéria para estudar a anatomia vascular da pelve (arteriografia pélvica panorâmica) e posteriormente cateterizar seletivamente as artérias uterinas com uso de contraste ${ }^{2,5,6}$.

Uma vez estando na artéria uterina, liberou-se o agente embolizante (polyvinyl alcohol-PVA, álcool polivinílico, ou embosferas com diâmetro de 500 a $900 \mu \mathrm{m})$, sob pressão baixa (e lentamente) até desaparecer o fluxo nos miomas. Depois é efetuado um controle arteriográfico final pós-embolização. O mesmo procedimento é feito na artéria uterina contralateral através da mesma punção. Estes aspectos, dos materiais e da técnica, são de extrema importância para minimizar os riscos de necrose maciça no útero e eventual insuficiência ovariana.

Do total de pacientes, em apenas seis foi usada analgesia parenteral e oral; as demais (106) pacientes foram submetidas a analgesia com morfina através de raquianestesia ou anestesia peridural para controlar a dor no pós-procedimento, o que foi efetivamente obtido.

Apenas nove $(8 \%)$ do total tinham desejo de engravidar, sendo que quatro delas o conseguiram. Duas $(22,2 \%)$ evoluíram para abortamento precoce (6 e 8 semanas de gestação) e outras duas (22,2\%) conseguiram parto a termo. Abaixo, relatamos estes dois casos.

\section{Caso 1:}

Primeira consulta em 26 de dezembro de 2003: Paciente com 35 anos, branca, nuligesta, 
apresentando leiomioma uterino diagnosticado há 4 anos em exame de rotina. No acompanhamento observou-se aumento progressivo no número e tamanho dos leiomiomas, evoluindo com aumento no fluxo menstrual e dor abdominal contínua, com piora no período menstrual. Apresentava também pressão no hipogástrio e polaciúria. Relatava uso de Lupron $\AA^{\circledR}$, por 6 meses, um ano antes do atendimento, com diminuição discreta e temporária no tamanho dos nódulos. Estava tentando engravidar a dois anos. Antecedente pessoal de hipotireoidismo, em uso de Synthroid, $75 \mathrm{mg} /$ dia.

A ressonância magnética nuclear pré-embolização mostrou volume uterino de $564 \mathrm{~cm}^{3}$, com 10 miomas intramurais distribuídos difusamente, sendo o maior com $70 \mathrm{~mm}$ na parede posterior.

A histeroscopia diagnóstica mostrou cavidade uterina aumentada e compressão extrínseca da parede posterior e óstio tubário prévios. A histerossalpingografia estava normal.

Foi realizada embolização das artérias uterinas em 21 de janeiro de 2004, usando-se, com êxito, partículas (500 a $700 \mu \mathrm{m})$ de álcool polivinílico. A paciente evoluiu bem, sem intercorrência, com alta hospitalar após $24 \mathrm{~h}$.

Três meses após a embolização os sintomas desapareceram e a paciente estava eumenorréica. O volume do útero reduziu para $34 \%\left(370 \mathrm{~cm}^{3}\right)$ no $3^{\circ}$ mês e $53 \%$ no $6^{\circ}$ mês $\left(265 \mathrm{~cm}^{3}\right)$. Observamos redução semelhante no nódulo maior. Em seguida a paciente engravidou espontaneamente, com gestação gemelar.

A gravidez evoluiu sem intercorrência e sem crescimento dos nódulos de leiomioma. Na trigésima sexta semana e 5 dias a paciente entrou em trabalho de parto, sendo realizado parto cesariana, sem intercorrência. A dequitação e o sangramento pós-parto foram normais. Na gravidez não houve sinal de restrição ao crescimento dos fetos, com evolução normal no volume de liquido amniótico e perfil hemodinâmico uterino, placentário e fetal. O peso dos recém-nascidos foi de 2.450 e $2.280 \mathrm{~g}$, e o puerpério transcorreu sem anormalidade.

\section{Caso 2:}

A primeira consulta ocorreu em 10 de novembro de 2000: Paciente com 34 anos, amarela, com leiomioma uterino há 2 anos, diagnosticado em exame de rotina, sendo que durante o acompanhamento houve aumento no número e tamanho dos miomas.

Os ciclos menstruais eram normais desde a menarca, sem modificação em suas características. Negava cólica menstrual ou sintoma compressivo. Nuligesta e no momento não tinha desejo de engravidar, mas necessitava tratar a doença para manter a capacidade reprodutiva futura.

A paciente foi orientada a utilizar Lupron ${ }^{\circledR}$ de fevereiro a junho de 2000, e em agosto de 2000 foi realizada videolaparoscopia cirúrgica para realizar miomectomia. Não houve sucesso pela dificuldade e risco de sangramento e de eventual histerectomia, sendo então encaminhada para embolização arterial.

Na ressonância magnética nuclear o volume do útero era de $385 \mathrm{~cm}^{3}$, com múltiplos miomas intramurais. A histeroscopia evidenciou cavidade endometrial aumentada, sem outra alteração.

No dia 01 de dezembro de 2000 foi realizada embolização nas artérias uterinas com partículas (300 a $500 \mu \mathrm{m})$ de álcool polivinílico, sem intercorrência. A paciente evoluiu bem e obteve alta hospitalar após $24 \mathrm{~h}$. Compareceu para acompanhamento rotineiro de 3 em 3 meses, sendo que aos 9 meses pós-embolização o volume do útero era de $132 \mathrm{~cm}^{3}$ (redução de 65\%) e os miomas foram reduzidos, em média, para metade do volume original. Estes aspectos radiológicos se mantiveram estáveis até o segundo ano de acompanhamento.

Em julho de 2004 (três anos e meio após a embolização) o volume uterino e o tamanho dos miomas permaneciam estáveis e a paciente engravidou espontaneamente. A gravidez transcorreu normalmente, sem aumento no tamanho dos miomas e sem comprometimento dos compartimentos hemodinâmicos feto-placentário. Em abril de 2005 a paciente estava com 39 semanas de gestação, tendo sido realizado parto cesariana. Durante o parto foi diagnosticado acretismo placentário, tendo sido realizada histerectomia subtotal, sem intercorrência. O peso do recém-nascido foi $3.370 \mathrm{~g}$.

\section{Discussão}

O tratamento do leiomioma uterino por embolização arterial vem sendo realizado há mais de 15 anos, estando consagrado como mais uma opção terapêutica para esta enfermidade ${ }^{7}$. Este procedimento proporciona ótimo resultado em relação ao controle dos sintomas (alterações menstruais, algia pélvica e sintomas compressivos) e uma significativa redução nos volumes do útero e dos miomas.

Meta-análises avaliaram os resultados de mais de 150 centros que realizam embolização nas artérias uterinas (mais de 80.000 pacientes) e referem que o controle clínico ocorre de 85 a $95 \%$ dos casos. A redução média no volume do útero é de 60 a 70\% do volume inicial, com diminuição de 40 a $60 \%$ no volume dos miomas ${ }^{8-12}$. 
O índice de satisfação das pacientes após o tratamento foi de aproximadamente 80 a $90 \%$, a taxa de recidiva (reincidência), menor que 10\%, o tempo médio de internação, de $24 \mathrm{~h}$, e o de retorno às atividades habituais, de 3 a 5 dias $^{8-12}$.

Inicialmente indicava-se este tratamento para pacientes com prole definida, pois não se conhecia o impacto da embolização arterial sobre a fertilidade. O que se temia era o eventual risco de necrose uterina maciça ou infecção uterina, com possivel necessidade de histerectomia. A ocorrência de endometrite ou piometrite é rara, não passando de $1 \%$ dos casos, e está associada ao uso de partículas da PVA muito pequenas (150 a $250 \mu \mathrm{m})^{2}$. Necessidade de histerectomia pós-embolização pode ocorrer em 1 a cada 700 pacientes tratados.

Outra situação controversa e polêmica é o risco para insuficiência ovariana prematura pósembolização. Sabe-se que está diretamente relacionado com a idade da paciente. Em mulheres com menos de 40 anos pode ocorrer em $0,7 \%$ dos casos, porém chega a atingir perto de $35 \%$ das mulheres com mais de 45 anos.

Uma condição que poderia explicar o risco de comprometimento da circulação ovariana seria quando a vascularização da gônada depende exclusivamente do ramo ovariano da artéria uterina. Esta situação ocorre em cerca de 4\% das mulheres. Diante desta preocupação realiza-se durante o procedimento da embolização um estudo da circulação do ovário e, dependendo do resultado, pode-se até interromper o procedimento se houver risco de obstrução da irrigação desta glândula.

Outro aspecto que pode estar envolvido no processo de comprometimento da circulação ovariana é o uso de partículas com diâmetro menor que $500 \mu \mathrm{m}$. Como o diâmetro do ramo ovariano da artéria uterina é menor que $500 \mu \mathrm{m}$, estas não obstruiriam esta circulação. Atualmente utilizam-se na rotina partículas com diâmetro maior, especialmente entre 700 e $900 \mu \mathrm{m}$ para se evitar este risco.

Supõe-se também que se houver obstrução troncular na artéria uterina ou se injetarmos o material embolizante com alta pressão pode-se determinar alterações na irrigação da gônada, sendo este um cuidado que precisamos ter durante o procedimento.

Com estas mudanças na técnica e no material utilizados durante o procedimento houve diminuição significativa nas complicações. Portanto, atualmente preconiza-se usar partículas com tamanho maior (geralmente acima de $700 \mu \mathrm{m}$ ) e ter cuidado com o ponto ideal para interromper o lançamento das partículas (end point), de modo a bloquear somente a circulação dos miomas, sem comprometer o tronco da artéria uterina ${ }^{3,4}$.
Diante destes dados pôde-se iniciar a indicação deste tratamento em pacientes que desejavam manter a capacidade reprodutiva, especialmente quando o mioma está associado com infertilidade e não é susceptível a outro tratamento cirúrgico conservador, ou quando há falha no tratamento conservador prévio ${ }^{13}$.

Antes de indicar qualquer conduta terapêutica é extremamente importante avaliar detalhadamente cada caso através dos aspectos clínicos, radiológicos e histeroscópicos, para determinar qual a opção terapêutica que trará os melhores resultados em termos de controle dos sintomas e miomas, e de fertilidade.

O relato da evolução de nossos casos é semelhante a várias descrições de séries com gravidezes e partos em mulheres submetidas a embolização arterial $^{12-16}$.

Os trabalhos que abordam a manutenção da fertilidade em pacientes submetidas a embolização referem que esta opção terapêutica é indicada em relação à miomectomia nos casos em que esta cirurgia não é factível, seja diante da dificuldade de realizá-la seja pelo risco de sangramento ou de eventual histerectomia. Também é indicada quando há recidiva do tumor. As seqüelas para a fertilidade, após miomectomias múltiplas ou que comprometem muito a parede uterina, seriam menores se fosse realizada uma embolização ${ }^{12}$.

Segundo meta-análises, a taxa de gravidez pode chegar a $40 \%$ dos casos após embolização em pacientes desejosas de gestação, o que é semelhante à taxa observada após miomectomia ${ }^{8,12}$.

Quanto aos resultados de gestações, não se observa aumento significativo nas complicações durante a gravidez, no parto e no puerpério, sendo concorde com o relato do nosso primeiro caso $^{12-14,16}$. É relatada uma freqüência significante de partos normais, em comparação com mulheres submetidas a miomectomia, sem aumento nas complicações, como rotura uterina ${ }^{13,14}$.

No entanto, pode ocorrer aumento na incidência de má apresentação fetal e parto prematuro quando comparada com gravidezes ocorridas após miomectomia laparoscópica, fato não descrito por outras referências e por nossa casuística. Já as taxas de aborto e hemorragia pós-parto foram semelhantes, tanto após a embolização como após a miomectomia ${ }^{15}$.

Acretismo placentário como ocorreu em nosso segundo caso também é referido em outros casos discutidos na literatura ${ }^{12}$. Outros dois casos apresentaram anormalidades placentárias, sem complicação no pós-parto. As alterações placentárias também têm incidência aumentada após miomectomia e cirurgias histeroscópicas, mas 
não há nenhum estudo comparando estas duas modalidades cirúrgicas com embolização ${ }^{17}$.

Diante destes dados, precisamos observar e analisar um número maior de pacientes que desejam engravidar, e que são submetidas a tratamento de mioma uterino por embolização das artérias uterinas, para podermos determinar vários aspectos ainda não esclarecidos. Também há necessidade de estudos randomizados comparando gestações após miomectomia e embolização ${ }^{18}$.

Conclui-se que a fertilidade pode ser mantida após o tratamento de leiomioma uterino por embolização das artérias uterinas, especialmente após algumas modificações na técnica e no material empregados no procedimento, minimizando ainda mais alguns receios que tínhamos em relação a necrose uterina e insuficiência ovariana prematura.

Todavia, o mais importante é analisar os aspectos clínicos, radiológicos e histeroscópicos de cada caso para depois indicar o melhor tratamento, promovendo, assim, melhores resultados clínicos e anatômicos, causando menor comprometimento à fertilidade e ao ciclo gravidico-puerperal.

\section{Referências}

1. Ravina $\mathrm{JH}$, Herbreteau D, Ciraru-Vigneron N, Bouret JM, Houdart E, Aymard A, et al. Arterial embolisation to treat uterine myomata. Lancet. 1995;346(8976):671-2.

2. Healey S, Buzaglo K, Seti L, Valenti D, Tulandi T. Ovarian function after uterine artery embolization and hysterectomy. J Am Assoc Gynecol Laparosc. 2004;11(3):348-52.

3. Richter GM, Radeleff B, Rimbach S, Kauffmann GW. Uterine fibroid embolization with spheric microparticles using flow guiding: safety, technical success and clinical results. Rofo. 2004;176(11):1648-57.

4. Goodwin SC, Vedanthan S, McLucas B, Forno AE, Perrella R. Preliminary experience with uterine artery embolization for uterine fibroids. J Vasc Interv Radiol. 1997;8(4):517-26.

5. Hutchins FL Jr, Worthington-Kirsch R, Berkowitz RP. Selective uterine artery embolization as primary treatment for symptomatic leiomyomata uteri: a review of 305 consecutive cases. J Am Assoc Gynaecol Laparosc. 1999;6(3):279-84.

6. Armstrong C, Caird L. Fibroid embolization: a technique not without significant complications. BJOG. 2001;108(1):132.
7. Spies JB, Cooper JM, Worthington-Kirsch R, Lipman $\mathrm{JC}$, Mills BB, Benenati JF. Outcome of uterine embolization and hysterectomy for leiomyomas: results of a multicenter study. Am J Obstet Gynecol. 2004;191(1):22-31.

8. Wong GC, Muir SJ, Lai AP, Goodwin SC. Uterine artery embolization: a minimally invasive technique for the treatment of uterine fibroids. J Womens Health Gend Based Med. 2000;9(4): 357-62.

9. Zhou SK, Li SB, Zhang ZS. Influential factors to therapeutic efficacy of uterine artery embolization in the treatment of uterine fibroids. Hunan Yi Ke Da Xue Xue Bao. 2003;28(4):409-11.

10.Jacob D, Rafii A. Embolization of uterine arteries for uterine fibroids: state-of-the-art. Gynecol Obstet Fertil. 2004;32(11):927-36.

11.Smith WJ, Upton E, Shuster EJ, Klein AJ, Schwartz ML. Patient satisfaction and disease specific quality of life after uterine artery embolization. Am J Obstet Gynecol. 2004;190(6):1697-703.

12.Pron G, Mocarski E, Bennett J, Vilos G, Common A, Vanderburgh L, et al. Pregnancy after uterine artery embolization for leiomyomata: the Ontario multicenter trial. Obstet Gynecol. 2005;105 (1):67-76.

13. Goodwin SC, Vedantham S, McLucas B, Forno AE, Perrella R. Preliminary experience with uterine artery embolization for uterine fibroids. J Vasc Interv Radiol. 1997;8(4):517-26.

14.McLucas B, Goodwin S, Adler L, Rappaport A, Reed R, Perrella R. Pregnancy following uterine fibroid embolization. Int J Gynaecol Obstet. 2001; 74(1):1-7.

15.Ravina JH, Vigneron NC, Aymard A, Le Dref O, Merland JJ. Pregnancy after embolization of uterine myoma: report of 12 cases. Fertil Steril. 2000;73 (6):1241-3.

16.Goldberg J, Pereira L, Berghella V, Diamond J, Darai E, Seinera P, et al. Pregnancy outcomes after treatment for fibromyomata: uterine artery embolization versus laparoscopic myomectomy. Am J Obstet Gynecol. 2004;191(1):18-21.

17.Carpenter TT, Walker WJ. Pregnancy following uterine artery embolisation for symptomatic fibroids: a series of 26 completed pregnancies. BJOG. 2005; $112(3): 321-5$.

18. Cook JL, Seman EI. Pregnancy following endometrial ablation: case history and literature review. Obstet Gynecol Surv. 2003;58(8):551-6. 